\title{
Double Chimeric Peptide Vaccine and Monoclonal Antibodies that Protect Against Disseminated Candidiasis
}

\section{Hong Xin *}

Department of Pediatrics, Louisiana State University Health Sciences Center and Research Institute for Children, Children's Hospital, New Orleans, Louisiana 70118, USA

“Corresponding author: Hing Xin, Research Institute for Children, Children's Hospital, 200 Henry Clay Ave, New Orleans, LA 70118, USA, Tel: (504) 896-2912; Fax: (504) 896-9413; E-mail: hxin@chnola-research.org

Received date: 22 May 2014; Accepted date: 26 June 2014; Published date: 30 June 2014

Copyright: (c) 2014 Xin H. This is an open-access article distributed under the terms of the Creative Commons Attribution License, which permits unrestricted use, distribution, and reproduction in any medium, provided the original author and source are credited.

\begin{abstract}
A double chimeric peptide vaccine, targeting two peptides (Fba and Met6) expressed on the cell surface of Candida albicans, can induce high degree of protection against disseminated candidiasis in mice. Although immunization with each individual peptide vaccine was effective in protection against the disease, immunization with the double chimeric peptide vaccine, not two- peptide mixture, induced better protective immunity than that induced by each individual peptide vaccine. Passive transfer of immune sera from peptide immunized mice demonstrated that the protection was medicated by peptide-specific antibodies. Furthermore, we combined two protective MAbs, including Fba peptide specific IgM E2-9 and Met6 peptide specific IgG3 M2-4, into one cocktail and evaluated it for protective efficacy, side by side with each protective MAb given alone. Survival data indicate that while the use of individual $\mathrm{MAb}$ is promising as a single prophylactic component, combination of two MAbs manifested the best protective efficacies. Our work demonstrates the novel utility of cell wall peptides together in vaccines designed for induction of greater protective immunity against disseminated candidiasis. We also show the combination of monoclonal antibodies is a more efficient immuneprotective approach against the disease as compared to single MAb treatment.
\end{abstract}

Keywords: Peptide vaccine; Antibody protection; Adjuvant; Candidiasis

\section{Introduction}

Hematogenously disseminated candidiasis in humans is the third leading cause of nosocomial bloodstream infection in the US. Despite the availability of appropriate antifungal therapy, crude mortality in the last decade has remained high, ranging from 36 to $90 \%$ [1-3]. To date there is no approved antifungal vaccine for use in humans and significant therapeutic challenges remain, which emphasizes the need for effective preventative strategies.

Candida albicans, which is the most prevalent fungal pathogen of humans, has involved a multitude of mechanisms to survive in the host. Due to the complexity of $C$. albicans antigens and invade mechanisms, it is difficult to obtain effective immune protection with a single antigen. Therefore, there is a need to combine two or more vaccine candidate antigens to develop multivalent vaccine for the best efficacy [4]. Previously we showed antibodies specific for the peptide Fba, which was derived from fructose-bisphosphate aldolase on the cell wall of $C$. albicans, protect mice against disseminated candidiasis [5,6]. In this study, another protective peptide Met6, which was derived from another $C$. albicans cell wall protein 5methyltetrahydropteroyltriglutamate homocysteine methyltransferase, was also able to induce protection against disseminated candidiasis by itself. Passive transfer of immune sera from Met6 peptide vaccinated mice conferred protection in naive mice. This result along with our finding that an IgG3 MAb M2-4, which is specific for the Met6 peptide, protected mice against candidiasis provide strong evidence that antibodies contribute to protection. Therefore, peptide Met6, which appears to be an effective carrier for the protective glycan epitope in a glycopeptide vaccine formulation [5], is also an excellent candidate by itself for vaccine development against candidiasis. To induce more effective protection, we combined the two protective peptides into a single double chimeric peptide vaccine against $C$. albicans. By a similar DC-based immunization approach favoring antibody production as we described before [5], we tested and compared efficacy of the double chimeric peptide vaccine with each individual peptide vaccine and the two-peptide mixture. The double chimeric peptide vaccine (Fba-Met6 conjugate) enhance survival most significantly, which is evidenced by $100 \%$ survival rate and much less reduced CFUs in kidneys of animals as compared to animals immunized with each individual peptide vaccine or peptide mixture. Furthermore, we also evaluated the protection of two protective MAbs, E2-9 and M2-4, given alone, or double-MAb combination (E2-9/ M2-4) in naïve mice. While the use of individual MAb (E2-9 or M2-4) is promising as single prophylactic component, combinations of MAbs (E2-9/M2-4) manifested the best protective efficacies. Our work demonstrates that development of a double chimeric peptide vaccine that induces protective immune responses against two unrelated cell surface peptide epitopes of $C$. albicans is a better approach against the disease. In addition, combinations of multiple MAbs may provide the most effective form of passive transfer protection, which may demonstrate emergent properties with regard to protective efficacy.

\section{Materials and Methods}

\section{Candida strains and culture conditions}

C. albicans 3153A and SC5314, were grown as stationary-phase yeast cells in glucose-yeast extract-peptone broth at $37^{\circ} \mathrm{C}$, washed and suspended to the appropriate cell concentration $\left(5 \times 10^{6} / \mathrm{ml}\right)$ in 
Dulbecco's PBS (DPBS; Sigma), and used to infect mice intravenously (i.v.) as described [7,8]. C. albicans strain 3153A was also used for serum antibody absorption, immunofluorescence staining and flow cytometric analysis.

Mice. BALB/c and C57BL/6 female mice (National Cancer Institute Animal Production Program, Frekerick MD) 5 to 7 weeks old were used throughout. Mice were maintained in our AAALAC-certified animal facility and all animal experiments were done in accordance with protocols numbers 180 approved by the Institutional Animal Care and Use committee (IACUC) at Children's Hospital Research Institute in New Orleans.

\section{Isolation and culture of dendritic cells (DCs) from mouse bone marrow}

Dendritic cells (DCs) were generated from mouse bone marrow by a previously described method [5,9]. Briefly, donor mice were euthanized by $\mathrm{CO}_{2}$ asphyxiation, their long bones and tibias were aseptically removed, bone marrow was flushed from the bones by forcibly injecting several $\mathrm{ml}$ of RPMI-1640 and clumps were removed or dispersed by gentle pipetting through a sterile $70-\mathrm{mm}$ cell strainer. Red blood cells were lysed (ACK lysing buffer, $0.15 \mathrm{M} \mathrm{NH}_{4} \mathrm{Cl}, 1.0 \mathrm{mM}$ $\mathrm{KHCO}_{3}, 0.1 \mathrm{mM}$ EDTA) for $4 \mathrm{~min}$ and the remaining bone marrow cells were suspended in complete medium [CM, RPMI-1640 supplemented with 10\% FBS (FBS), $2 \mathrm{mM}$ L-glutamine, $1 \%$ of nonessential amino acids and 100 units $/ \mathrm{ml}$ penicillin and $100 \mu \mathrm{g} / \mathrm{ml}$ streptomycin], adjusted to $2 \times 10^{5}$ cells per ml plated in 6-well plates at $5 \mathrm{ml}$ per well and cultured for up to 9 days in the presence of $40 \mathrm{ng} / \mathrm{ml}$ of rmGM-CSF and rmIL-4 (R\&D Systems) at $37^{\circ} \mathrm{C}, 5 \% \mathrm{CO}_{2}$. On days 4 and 7 of culture, the same amount of fresh GM-CSF and IL- 4 was added to the wells.

\section{Peptide vaccine, peptide-8MAP and double chimeric peptide vaccine}

Two 14-mer peptides Fba and Met6 are derived from C. albicans cell wall proteins fructose-bisphosphate aldolase (Fba) and methyltetrahydropteroyltriglutamate (Met6). Fba peptide and Met6 peptide were each conjugated to a multiple antigenic peptide (MAP), of which the lysine core displayed approximately eight copies of the peptide epitope, to form Fba-8Map and Met6-8MAP. Fba peptide (YGKDVKDLFDYAQE), Met6 peptide (PRIGGQRELKKITE), Fba-8MAP peptide, Met6- 8MAP peptide and Fba-Met6 double chimeric peptide (YGKDVKDLFDYAQE-KK- PRIGGQRELKKITE ) in which two peptides were joined by a lysine linker (-KK-) were produced commercially (GenScript).

Peptide Immunizations with peptide pulsed dendritic cells. DCs were pulsed in vitro with Fba peptide, Met6 peptide, Fba-Met6 double chimeric peptide and peptides Fba/Met6 mixture as described before with modification by totally removing CFA for last booster (5). Briefly, DCs in culture were pulsed with the peptide antigen $(1 \mu \mathrm{M})$ on day 6 . On day 7, PGE2 (10-7 M) was added along with LPS ( $2 \mu \mathrm{g} / \mathrm{ml}$, Sigma) for $24 \mathrm{~h}$. On day 9, antigen-pulsed DCs were washed extensively and $5 \times 105$ in $200 \mu \mathrm{l}$ DPBS were given i.p. as the priming dose to mice. The mice were boosted i.p. at day 14 and day 28 with fresh antigen-pulsed DCs without adjuvant.

\section{Immunizations with peptide vaccine in human approved adjuvant Alum}

Fba-8MAP and Met6-8MAP peptides were administered as a mixture made with alum (aluminum hydroxide gel, Sigma) as adjuvant. Mice were immunized by s.c. injection $100 \mu \mathrm{l}$ of $2.5 \mu \mathrm{g}$ of each peptide- $8 \mathrm{MAP}$ conjugate (Fba-8MAP or Met6-8MAP) with 50 $\mu \mathrm{g}$ alum on days 1,21 and 42 . Sera from groups of mice given DPBS buffer or adjuvant only were used as negative controls.

\section{Serological assays}

Sera were ELISA analyzed for antibody titers. For DC-based immunization, control groups consisted of mice given DCs alone, or DPBS alone for all three injections. For Fba-8MAP or Met6-8MAP peptide administered with alum, control groups were mice given adjuvant alone or DPBS buffer. Fba-8MAP or Met6-8MAP (GenScript) was dissolved at $5 \mu \mathrm{g} / \mathrm{ml}$ in PBS (pH 7.4) and used to coat 96-well ELISA plates for testing duplicate serial 2-fold dilutions of samples of each immune serum and control sera. Color development for each well was achieved by use of secondary antibody (goat antimouse polyvalent Ig-HRP, Sigma) and substrate ( $O$-phenylenediamine and $\mathrm{H} 2 \mathrm{O} 2$ ) and $\mathrm{OD}$ determined at $492 \mathrm{~nm}$.

\section{MAbs}

Hybridoma clones, which produce Fba peptide specific MAb IgM E2-9 [6] and Met6 peptide specific MAb IgG3 M2-4, were generated from mice vaccinated with Fba-DC or Met6-DC preparation as described previously [5]. Briefly, BALB/c mice were immunized by injection of synthetic peptide pulsed DCs to stimulate the production of antibodies against peptide as described above. Ten days after the second booster, serum was taken from each animal to determine animals with the highest anti-peptide titers for subsequent sacrificing, removal of spleens and preparation of single cell suspensions. Hybridoma clones were established by the polyethylene glycol facilitation of fusion of spleen cells to an SP2/0-AG1myeloma cell line by standard protocols. Hybridoma clones were screened by ELISA for production of specific anti-peptide antibody; only the highest titers and most rapidly growing clones were selected for subsequent cloning $\mathrm{x} 3$ or more by limiting dilution.

The hybridoma cell lines were initially grown in antibiotic-free RPMI 1640 medium (Sigma) supplemented with 10\% fetal bovine serum (Invitrogen) and $2 \mathrm{mM} \mathrm{L-glutamine} \mathrm{(Sigma)} \mathrm{at} 37^{\circ} \mathrm{C}$ and in the presence of $5 \% \mathrm{CO}_{2}$. For antibody production, the hybridoma clones were grown in antibiotic-free, BD cell MAb serum-free medium (but containing $1.1 \mathrm{mg}$ bovine serum albumin/ml) in a CELLine device (BD, Bedford, MA). The supernatant was collected and MAb was purified by affinity chromatography using a Protein A Sepharose 4FF column (GE Healthcare, USA). The isotype of mAb was determined with a Mouse Monoclonal Antibody Isotyping Kit (Pierce, USA).

\section{Fungal challenge and assessment of protection}

Two weeks after the second boost, immune and control mice were infected i.v. with a lethal dose of live $C$. albicans yeast cells $\left(5 \times 10^{5}\right.$ in $0.1 \mathrm{ml}$ of DPBS) prepared as described above and as before [5]. Passively immunized mice (below) also received the same challenge dose. Protection was evaluated by monitoring animal survival for 50-60 days. The mice were monitored for the development of a moribund state, defined as being listless, disinterested in food or water, 
and nonreactive to finger probing. At the time that a mouse was deemed moribund, it was sacrificed and their kidneys (for some experiments, brains) were homogenized in DPBS and plated onto a nutrient agar to determine colony forming units (CFUs).

\section{Passive transfer of MAbs by intraperitoneal (i.p.) route}

The preventive effect of MAbs M2-4 and E2-9 was examined by passive transfer experiments. The MAb E2-9 was appropriately diluted in DPBS $(0.2 \mu \mathrm{g} / \mu \mathrm{l})$ to give a 100,000 ELISA titer against Fba-MAP peptide coated on the plate, and the MAb M2-4 was appropriately diluted in DPBS $(0.5 \mu \mathrm{g} / \mu \mathrm{l})$ to give a 100,000 ELISA titer against Met6MAP peptide coated on the plate. For testing, mice received $0.5 \mathrm{ml}$ of MAb M2-4 or MAb E2-9 or $1 \mathrm{ml}$ of both MAbs in combination (0.5 $\mathrm{ml}$ of each) intraperitoneally. The negative control materials tested in mice were MAbs absorbed with $C$. albicans yeast cells (3153A) and DPBS. Control mice received $0.5 \mathrm{ml}$ of the DPBS diluents or adsorbed MAb solution. For each condition, 6- to 8-week-old female BALB/c mice (NCI) were given $0.5 \mathrm{ml}$ of test $\mathrm{MAb}$, or control materials intraperitoneally, followed $4 \mathrm{~h}$ later by $0.1 \mathrm{ml}$ intravenously of a suspension containing $5 \times 10^{6}$ yeast cells per milliliter of DPBS. Mice were divided into groups with five mice each and three independent experiments were carried out. In some experiments, for MAb M2-4 or MAb E2-9 group, mice were given the same dose of antibodies (MAb E2-9 or MAb M2-4) or control materials on every other day postchallenge for two weeks. All mice were sacrificed on day 50 .

\section{Statistical analysis}

Survival times were statistically evaluated by Kaplan-Meier (GraphPad Prism, version 4). In all analyses, there were five mice per group $(n=5)$ and a two- tailed $t$ test was used.

\section{Results}

Vaccination with Met6 peptide induced antibody production and protection against disseminated candidiasis in mice. C. albicans cell surface peptides were selected from previously identified cell wall proteins that are expressed during pathogenesis of human disseminated candidiasis $[10,11]$. Among the six peptide epitopes, we first focused on peptide Fba, which turned out to be an excellent peptide vaccine against $C$. ablicans infection [6]. When used alone to pulse DCs for subsequent immunizations, peptide Fba induced robust protective responses, which was medicated by Fba peptide-specific antibodies [6]. By the same antigen-pulsed DC-based immunization strategy favoring production of antibodies as described before [5,9], another cell surface peptide Met6 also induced good protection as evidenced by survival (Figure 1A) and reduced or non-detectable fungal burden (colony forming units, CFUs) in kidney (Figure 1B), a target organ in disseminated candidiasis, in mice challenged with the fungus. Antibody activity specific for the Met6 peptide appears to be responsible for the anti-Candida protection, as was demonstrated by experiments involving passive transfer of whole immune serum from Met6 peptide vaccinated mice, which conferred protection to naïve mice (Figure 1C). Such protection was not conferred by control DPBS buffer and the protection was abrogated when immune serum was pre-absorbed by fungal cells prior to the passive transfer protection test (Figure 1C).

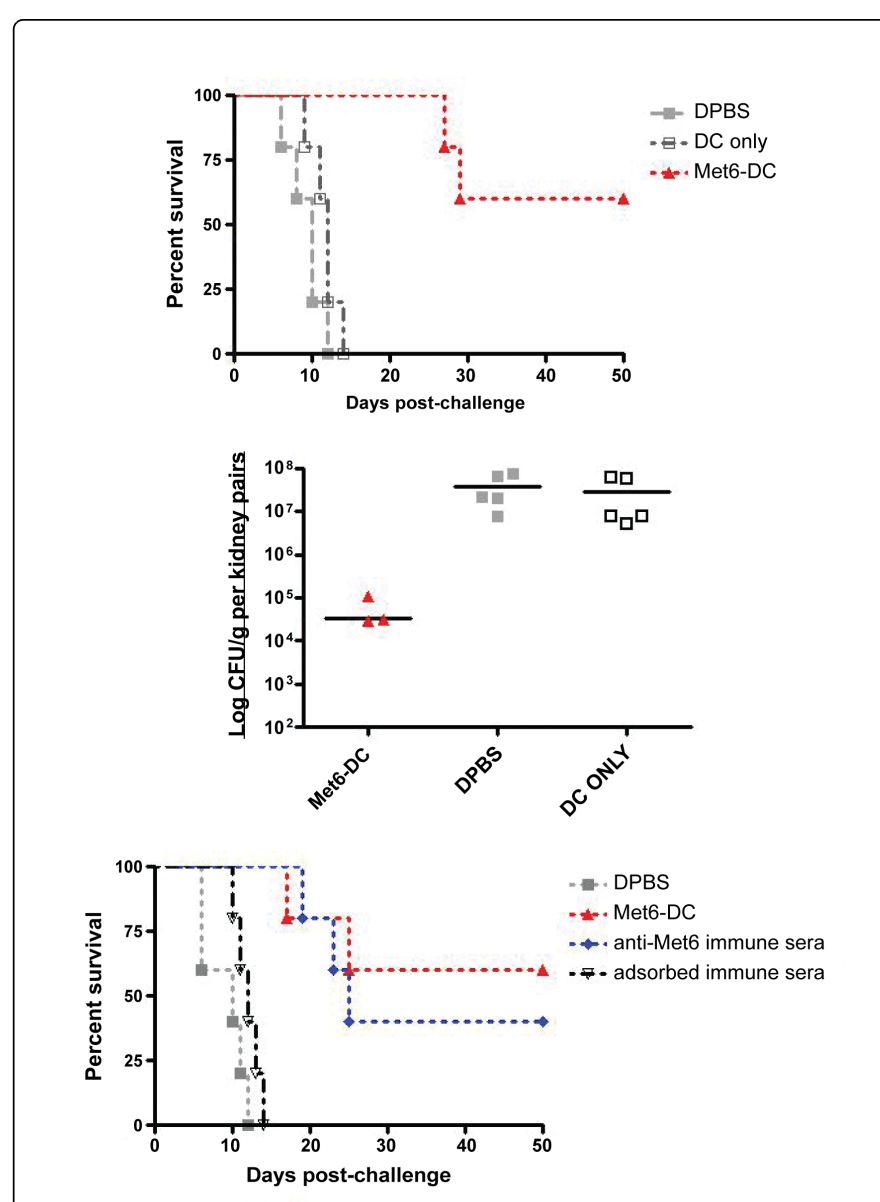

Figure 1: Met6 peptide vaccines induce protective responses in mice against disseminated candidiasis. Vaccination with Met6 peptide by the dendritic cell approach induced significant protection against experimental disseminated candidiasis in mice, as compared to control groups. (A) Vaccinated mice challenged with a lethal dose of a prototypical strain of C. albicans (SC5314) had a significant prolonged survival time as compared to control mice that received DCs or DPBS $(\mathrm{P}<0.01)$. (B) Consistently with survival data, immunized mice had greatly reduced or non-detectable CFUs in their kidneys $(\mathrm{P}<0.001)$ as compared to control mice. (C) Antibody is responsible for protection against disseminated candidiasis. Enhanced protection against disseminated C. albicans infection was observed in mice that received serum from mice immunized with the Met6 peptide as compared to animals that received control materials. Note that the mice immunized with Met6 peptide, which were used as positive controls for protection, had similar survival curve as the naïve mice that received the immune serum. Importantly, absorption with C. albicans before transfer also removed the protective value of immune serum.

Vaccination double peptide mixture didn't improve antibody production and protection against disseminated candidiasis in mice. To induce more effective protection, the peptide Fba was mixed with peptide Met6 to form double-peptide mixture for immunization in mice. By the similar dendritic cell (DC)-based approach, we tested each individual peptide, Met6 and Fba, side by side with peptide mixture for vaccine efficacy. Immunized groups that received the 
peptide Fba, peptide Met6 or Fba-Met6 mixture vaccines showed $60-80 \%$ survival throughout the 50 days post-challenge observation period and survived significantly longer as compared to DPBS or DC only controls following the lethal challenge (Figure 2A). Peptide or peptide mixture immunized groups had greatly reduced or even nondetectable viable fungal CFUs in kidneys (Figure 2B) as compared to control animals $(\mathrm{p}<0.0001)$. However, The Fba/Met6 peptide mixture didn't increase protective efficacy as compared to that induced by each individual peptide vaccine alone (Figure 1A), and Fba/Met6 mixture groups didn't show significantly reduced CFUs in kidneys as compared to individual peptide groups (Figure 2B). There is no significant difference in peptide-related antibody responses between Fba/Met6 mixture group and each individual peptide group (data not shown).
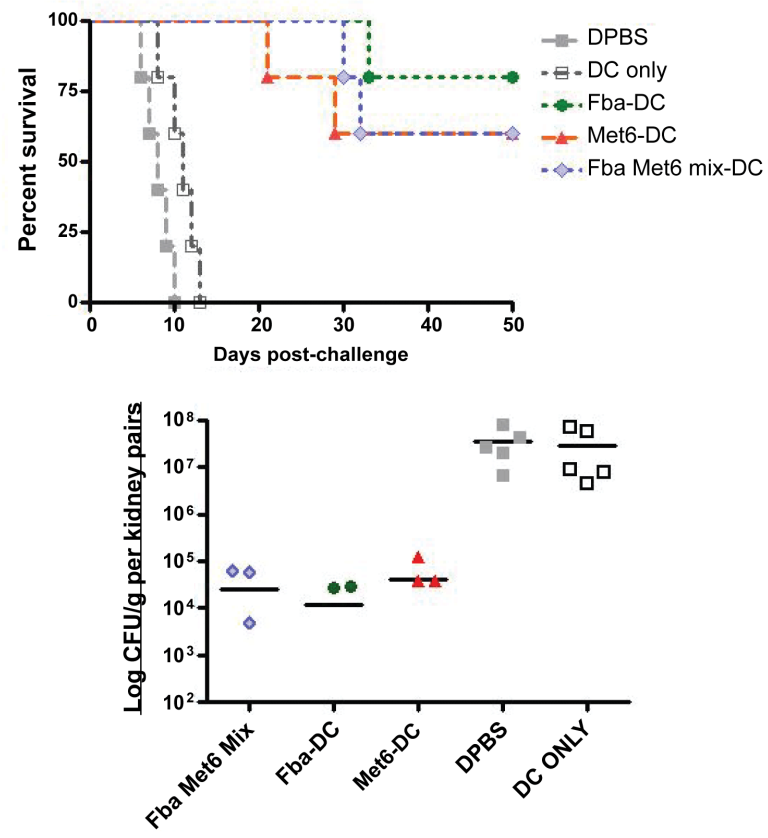

Figure 2: Vaccination with double peptide mixture didn't improve antibody production and protection against disseminated candidiasis in mice. By the dendritic cell (DC)-based approach, the peptide Fba was mixed with peptide Met6 to form double-peptide mixture for immunization in $\mathrm{BALB} / \mathrm{c}$ mice; peptide mixture induced protection was compared with each individual peptide vaccine. (A) Immunized groups that received the peptide Fba, peptide Met6 or Fba/Met6 mixture vaccines showed $60-80 \%$ survival throughout the 50 days post challenge observation period and survived significantly longer as compared to DPBS or DC only controls following the lethal challenge. Peptide Fba induced best protection. (B) Peptide or peptide mixture immunized groups had greatly reduced or even non-detectable viable fungal CFUs in kidneys as compared to control animals $(\mathrm{p}<0.0001)$. However, Fba/ Met6 mixture group didn't show significantly reduced CFUs in kidneys as compared to individual peptide groups. There is no significant difference in peptide-related antibody responses between Fba/Met6 mixture groups and each individual peptide group (data not shown).
Peptides Fba-8MAP and Met6-8MAP administered along with alum induced only modest protection against candidiasis. By use of the powerful DC-based immunization approach to overcome the weak immune responses of small molecules, the small synthetic peptide Fba and peptide Met6 vaccines were able to provide protection against disseminated candidiasis in mice [12]. However, the immunization approach could be inappropriate for human use, due to the complicated procedure and potentially expensive cost. To resolve this problem, Fba and Met6 peptide was each conjugated to multiple antigenic peptide (MAP) system, of which the lysine core displayed approximately eight copies of the peptide epitope, for immunization. To move toward a peptide vaccine against disseminated candidiasis more acceptable for human use, Fba-8MAP and Met6-8MAP each was administered as a mixture with alum (aluminum hydroxide gel, Sigma). Mice were immunized by subcutaneous (s.c.) injection of 0.1 $\mathrm{ml}$ containing $2.5 \mu \mathrm{g}$ of Fba-8MAP or Met6-8MAP mixed with $50 \mu \mathrm{g}$ alum on days 1,21 and 42. Negative control groups of mice were given a same volume of DPBS buffer or alum adjuvant only. Groups immunized with peptide pulsed DCs were used as positive controls. Serum samples were collected 14 days after immunization, diluted 1:200 and tested by ELISA on plates coated with synthetic peptide-8MAP. After the first booster, immune sera from mice immunized with Fba or Met6 peptide prepared in alum showed that antibody responses to each preparation peptide were about 4-5 fold greater over background sera obtained from mice that received DPBS or adjuvant only. However, the peptide specific antibody responses were relatively weaker as compared to that induced by peptide pulsed DC immunization approach (Figure 3A). Following the second booster immunization, an isotype switch from IgM to IgG of Fba and Met6 specific antibodies were observed in the sera from both peptideDC and peptide-8MAP immunized mice (data not shown), which suggested induction of an immune memory response. After challenge with a lethal dose of live $C$. albicans cells (3153A), the groups vaccinated with Fba-8MAP or Met6-8MAP mixed with alum had prolonged survival times as compared to two control groups; however the protection was not as strong as that induced by the peptide pulsed DC approach. Mice immunized with Fba-8MAP or Met6-8MAP administered along with alum had only $40 \%$ survival, while the groups immunized with peptide pulsed DC had better survival- $60-80 \%$ up to 50 days (Figure $3 \mathrm{~B}$ ). As expected, along with prolonged survival times, all the immunized groups had reduced live fungal cells in their kidneys as compared to controls. Fba-DC and Met6-DC groups had the least CFUs in kidneys among all the vaccinated groups (Figure 3C).

Double chimeric peptide pulsed DC vaccination induced solid protection against disseminated candidiasis in mice. As compared to single peptide vaccine, double peptide epitope vaccine may have more advantages, such as increased potency and breadth in protection. Since Fba and Met6 double-peptide mixture didn't induce better protection as compared to that induced by each individual peptide, we then used a different strategy by conjugating two peptides into one chimeric vaccine. Fba peptide was conjugated to the N-terminus of Met6 peptide through double lysine linker (-KK-), which is the target sequence of the lyzosomal protease cathepsin $\mathrm{B}$ for antigen processing in the context of MHC-II antigen presentation [13,14], to form FbaMet6 double chimeric peptide vaccine. Since Fba peptide consistently induced best protection by DC-based immunization, mice immunized with Fba-DC were used as positive control to compare the efficacy between Fba peptide vaccine and Fba- Met6 chimeric peptide vaccine. After animals were challenged, similar protection patterns were observed in all the immunized mice (Figure 4A). 

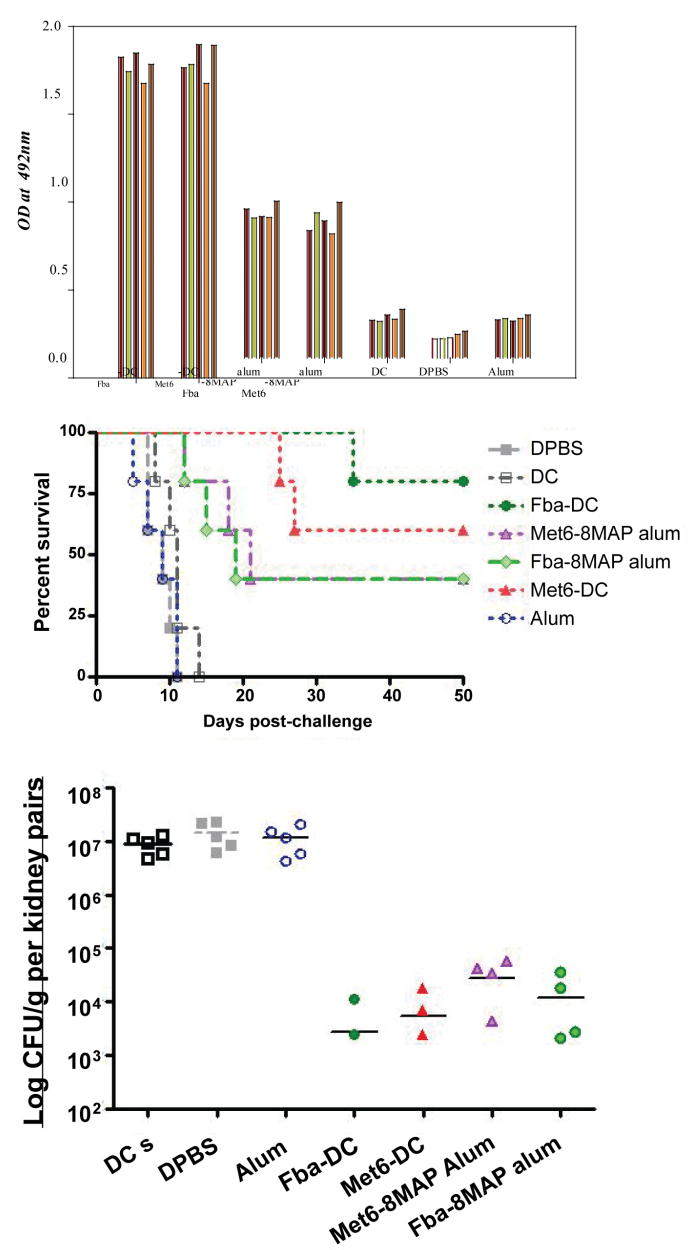

Figure 3: Peptides Fba-8MAP and Met6-8MAP administered along with the human approved adjuvant alum induced modest protection against candidiasis. (A) Serum samples were collected 14 days after immunization, diluted 1:200 and tested by ELISA on plates coated with synthetic Fba-8MAP and Met6-8MAP. After the first booster, immune serum from mice immunized with peptide-8MAP prepared in alum showed that antibody responses to the peptides Fba or Met6 were about 4-5 fold greater than that of sera from groups that received DPBS or adjuvant only. However, the peptide specific antibody responses induced by peptide- MAP system were relatively weaker as compared to that induced by peptide pulsed DC immunization approach. (B) After challenge with a lethal dose of live $C$. albicans cells (3153A), the groups vaccinated with Fba-8MAP or Met6-8MAP mixed with alum had prolonged survival times as compared to two control groups $(\mathrm{p}<0.01)$; however, the protection was not as strong as that induced by the peptide pulsed DC approach. (C) Being consistent with prolonged survival times, all the immunized groups had reduced live fungal cells in their kidneys as compared to controls $(\mathrm{P}<0.001)$. Fba-DC and Met6-DC groups had the least CFUs in kidneys among all the vaccinated groups.

In addition to prolonged survival times, each immunized group had reduced or non-detectable CFUs in their kidneys as compared to nonimmune mice (Figure 4B). Impressively, Fba-Met6 chimeric vaccine induced $100 \%$ complete protection up to 60 days when the experiment was terminated (Figure 4A), and only one mouse in that group had detectable low CFU in her kidneys (Figure 4B). Our data indicate FbaMet6 double chimeric peptide vaccine performed in a superior fashion as compared to other individual peptides or peptide mixtures. We also tested all the related peptide vaccines in C57BL/6 mice, which are more prone to Th1 responses, and obtained similar results (data not shown).

Combined IgG3 MAb (M2-4) and IgM MAb (E2-9) conferred enhanced protection against systemic candidiasis in passive transfer experiments. A monoclonal antibody (MAb IgG3 M2-4) specific for Met6 peptide was obtained by use of standard hybridoma techniques. Development of monoclonal antibodies specific for Met6 peptide affords us not only with important probes to study fungal expression of the peptide, but also provides us with the possibility of producing an unlimited supply of protective antibody for in vivo applications. First, MAb M2-4 was detected by an indirect immunofluorescence antibody test to confirm its specific reactivity with Met6 peptide on the cell surface of $C$. albicans (data not shown), and then BALB/c mice were given an i.p dose of MAb M2-4 four hours before hematogensous challenge with a lethal dose of $C$. albicans 3153A. Protective IgM MAb E2-9 specific for Fba peptide, which was isolated by us before [6], was used as positive control. Mice received either MAb M2-4 or MAb E2-9 had prolonged survival as compared to control animals that received either DPBS or adsorbed MAbs (Figure 5A); surprisingly, the MAbs combination (M2-4/E2-9) treatment was able to provide the best protection, to protect the naive mice completely (100\% survival). Consistently, the group received treatment of two protective MAb in combination had the least CFUs in kidneys among all the groups (Figure 5B). To test how many doses of each MAb are needed for the complete protection in naïve mice, each MAb was further evaluated by giving to naïve mice every other day post challenge for two weeks. Interestingly, when either MAb M2- 4 or E2-9 was given once, either MAb was able to provide $60 \%$ protection of recipient animals; however, when each MAb was given every other day for two weeks, MAb M2-4 was able to increase protection from $60 \%$ to $80 \%$, and E2-9, from $60 \%$ to $100 \%$. Two-week treatment with MAb E2-9 eventually reached the same protection provided by giving two-MAb combination once. Obviously, combination of two protective MAbs provides the best protection with reduced dose of each MAb administered. In all the transfer experiments, passive protection was prevented by removal of the MAbs through absorption with C. albicans cells before transfer (data not shown), which provided strong additional evidence for the protection being due to the MAbs.

\section{Discussion}

We originally began an investigation into the use of $C$. albicans cell wall derived peptides to serve as carrier for the small molecular weight glycan $\beta$-1,2-mannotriose, which we had demonstrated as a cell surface epitope inducing protective antibodies against disseminated candidiasis (14). Surprisingly, the peptide epitopes also induced antibody responses, and three peptides, including Fba, Met6 and Hwp1, induced protective responses against candidiasis when used alone as immunogen [5]. This lead to the current work in which the peptides Fba and Met6 were fused into one Fba-Met6 chimeric peptide vaccine that was characterized as novel bivalent peptide vaccine.

Synthetic peptide can be assembled into a single molecule (chimeric peptide) or just mechanically mixed. Formation of a single immunogenic construction from experimentally determined B- and T- 
epitopes provides optimal recognition of the components of this construction by the immune system [15]. In our study, to increase vaccine efficacy by inducing dual antibody-dependent protective immunity, the peptide Fba was first mixed with peptide Met6, and the peptide mixture was used to pulse DC for the subsequent immunization in mice. The Fba/Met6 mixture didn't increase protective efficacy as compared to that induced by each individual peptide. Fba peptide was then conjugated to peptide Met6 to form a double chimeric peptide (Fba-Met6) peptide vaccine, which induced complete protection that is more efficient than each individual peptide or peptide mixture. These results showed that a double chimeric peptide vaccine can induce better protective immunity than a single protective peptide epitope, which provided a feasible approach for developing a more effective peptide vaccine against candidiasis. In addition, as compared to simply mixed peptide mixture, conjugation of two distinctive peptides from different antigens may increase the chemical stability of peptides which can nonspecifically or specifically activate certain stages of the immune response to peptides and therefore potentiate this response [16]. On the other hand, synthetic peptide vaccines based on cytotoxic and Th1-epitopes represent mechanical mixtures of peptides corresponding to various T-epitopes from one or several antigens of an infectious agent [17]. Therefore, the design of monomolecular constructs is not required because in contrast to the B-T-epitope construction these T-epitopes can independently bind to various cells of the immune system.

Why does double chimeric peptide vaccine work better as a vaccine against $C$. albicans as compared to a single peptide vaccine? As compared to single peptide vaccine, double chimeric peptide vaccine has many advantages, including increased potency and breadth (18). Up to date, epitope-based vaccines not only focus the immune response on the effective protective motif of the target antigen, but they also allow for the construction of a chimeric vaccine by engineering multiple effective epitopes from different candidate antigens, thereby improving the level of protection in addition to avoiding immune evasion [18]. In our study reported here, the combination of unrelated protective peptide epitopes provides promising subunit vaccine constructs, with improved efficacy as compared to the individual peptide vaccine. The double chimeric peptide vaccine may also lead to the efficient induction of a wider range of antibody subclasses, which may explain the complete protection in vaccinated mice. These findings are promising for future immunization of genetically diverse human populations. The double chimeric peptide vaccine in this study can be a starting point for the potentially multivalent vaccine as a novel approach for developing more effective vaccines against fungal infections.
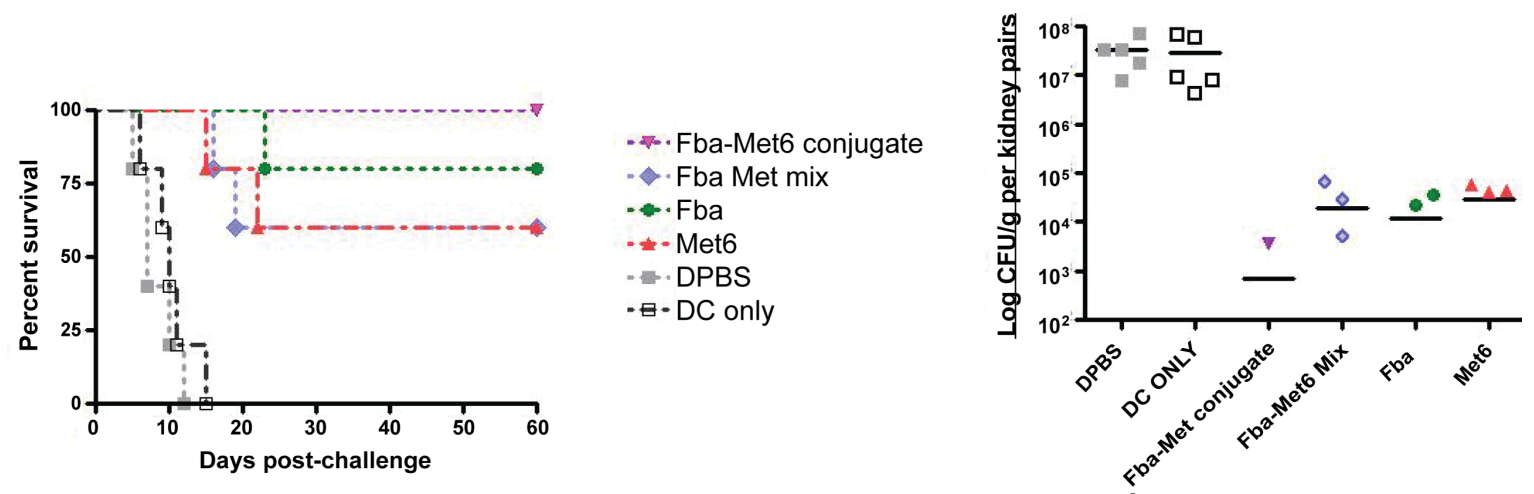

Figure 4: Double chimeric peptide pulsed DC vaccination induced solid protection against disseminated candidiasis in mice. Fba peptide was conjugated to the $\mathrm{N}$ terminus of Met6 peptide through double lysine linker (-KK-) to form Fba-Met6 double chimeric peptide vaccine. Mice immunized with Fba-DC and Met6-DC were used as positive controls to compare the efficacy between peptide vaccine and Fba-Met6 chimeric peptide vaccine. (A) After animals were challenged, similar protection patterns were observed in all the immunized mice. Impressively, Fba-Met6 chimeric vaccine induced $100 \%$ complete protection up to 60 days when the experiment was terminated. Our data indicate Fba-Met6 double chimeric peptide vaccine worked superior as compared to other individual peptide or peptide mixture. (B) In addition to prolonged survival times, each immunized group had reduced or non-detectable CFUs in their kidneys as compared to nonimmune mice. Only one mouse in Fba-Met6 conjugate group had detectable low CFU in her kidneys. We also tested all the related peptide vaccines in C57BL/6 mice, which are more prone to Th1 responses, and obtained the similar results (data not shown).

The multiple antigen peptide (MAP) system has long been proposed as a valuable approach for eliciting antibodies to peptides and developing synthetic vaccines [19]. To move toward to a more practical immunization approach for human use, Fba-8MAP and Met6-8MAP were tested and compared to peptide-pulsed DC based method. The ability of MAP system to elicit protective levels of antibody without the use of a carrier protein would be a distinct advantage in the development of synthetic peptide vaccines. However, due to the complexity of peptide-MAP system, the antibodies elicited by the octamer may differ significantly in their specificities as compared to singe peptide vaccine, which may explain the weaker antibody responses and reduced protective efficacy induced by the peptide-MAP system. However, an interesting feature of the MAP peptides is their ability to elicit good levels of antibodies following two immunizations with aluminum hydroxide, the only adjuvant currently licensed for use in human. It clearly offers the opportunity to design totally synthetic peptide vaccine, avoiding the requirement for carrier coupling, as well as allow for use human approved adjuvant. It has been reported that dimer or tetrameric structure of MAP system may improve the homogeneity of the elicited antibodies due to the reduced complexity of the peptide structure [19]. Further investigation is 
Citation: Xin H (2014) Double Chimeric Peptide Vaccine and Monoclonal Antibodies that Protect Against Disseminated Candidiasis . J Vaccines

needed for optimal responses induced by peptide-Map system (Figure

$5 \mathrm{C})$.
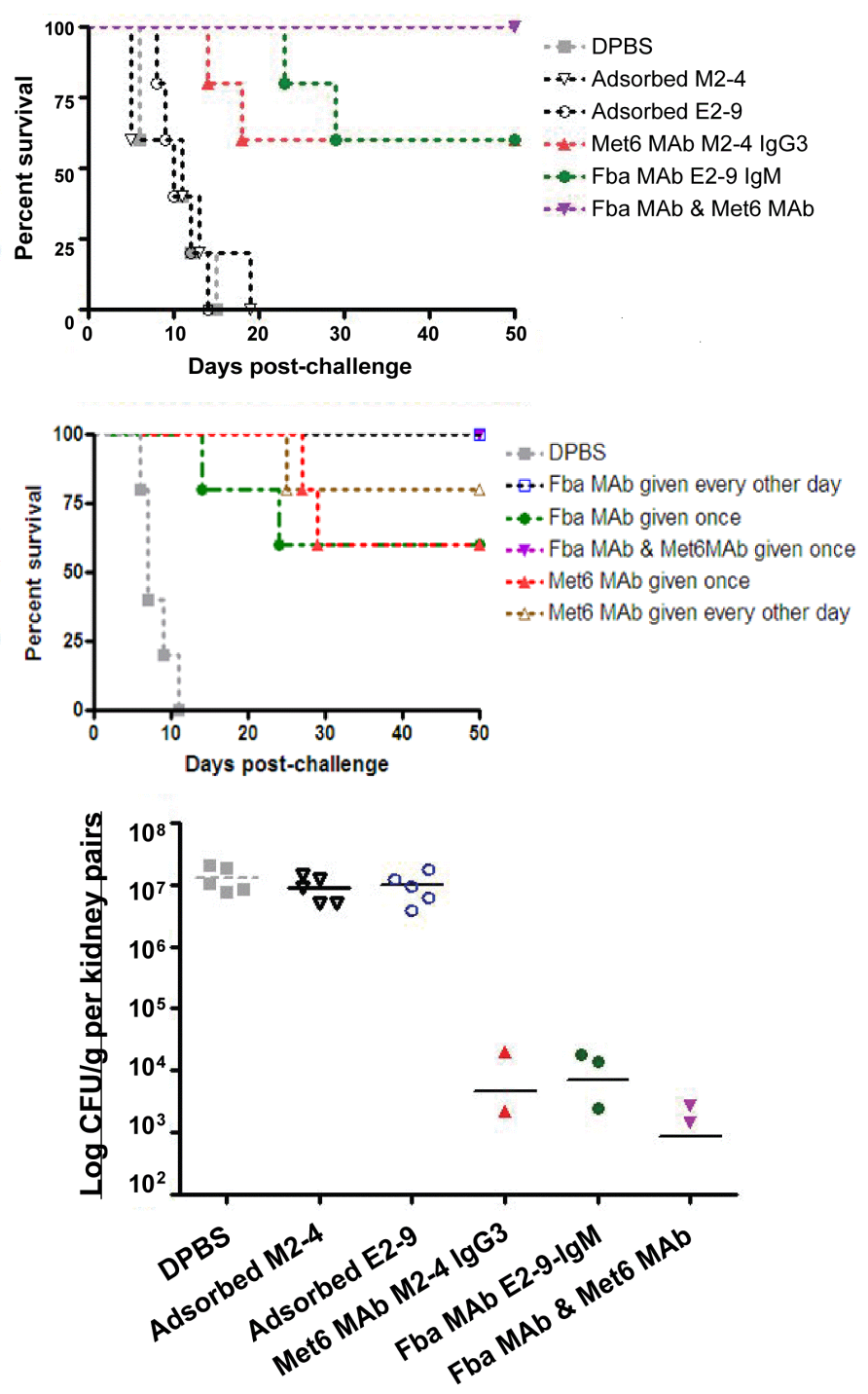

Figure 5: MAbs M2-4 and E2-9 in combination conferred best protection against systemic candidiasis in passive transfer experiments. A monoclonal antibody (MAb IgG3 M2-4) specific for Met6 peptide was obtained by use of standard hybridoma techniques. BALB/c mice were given an i.p. dose of MAb M2-4, or MAb E2-9 or the MAbs in combination four hours before hematogensous challenge with a lethal dose of C. albicans 3153A cells. (A) Mice received either MAb M2-4 or MAb E2-9 had 60\% survival rate with prolonged survival as compared to control animals that received either DPBS or adsorbed MAbs; surprisingly, the MAbs combination (M2-4/E2-9) treatment was able to provide the best protection, $100 \%$ survival up to 50 days. Passive protection was prevented by removal of the MAbs through absorption with $C$. albicans cells before transfer, which provided strong additional evidence for the protection being due to the MAbs. (B) Consistently, the group received treatment of two protective MAbs in combination had the least CFUs among all the groups $(\mathrm{P}<0.01)$. (C) To test how many doses of each MAb are needed for providing complete protection in naïve mice, each MAb was further evaluated by giving to naïve mice every other day post-challenge for two weeks. When given every other day for 14 days, MAb M2-4 was able to increase protection of recipient animals from $60 \%$ to $80 \%$, and for MAb E2-9, from $60 \%$ to $100 \%$. Combination of two protective MAbs provides the best protection with reduced dose of each MAb administered.

A possible limitation to the use of vaccines in immunosuppressed patients is that these patients may not necessarily mount protective responses, but passive immunization with protective antibodies may well be a rapid and effective preventive or even therapeutic measure
[20-22]. Over the past two decades, passive prophylactic or immuntherapeutics have become an increasingly attractive approach to combat infectious diseases. MAbs have the advantage over polyclonal reagents in that they are specific; have high activity per 
protein amount since all immunoglobulins bind to the intended target and can be produced in virtually unlimited amounts [23]. In addition to MAb E2-9 protects against disseminated candidiasis in mice, another protective MAb IgG3 M2-4, which is specific for the Met6 peptide, was also demonstrated in vivo in this study. When administered at a dose of $0.5 \mathrm{ml}$ (titer 1:100,000) $4 \mathrm{~h}$ before lethal challenge with $C$. albicans cells, MAb M2-4 provided significantly prolonged survival times, along with significant reduction in the number of CFUs in kidney from infected recipient mice as compared to mice that received buffered saline in place of the MAb $(\mathrm{p}<0.0001)$. In conclusion, loss of protective capacity of immune sera upon absorption with fungal cells and the passive protection conferred by MAb M2-4 provide strong evidence that anti-Met6 antibodies could provide an effective prevention or therapy for disseminated candidiasis. Although passive immunization of MAb against either peptide Fba or peptide Met6 has been successful in protecting mice against subsequent challenge with lethal dose of live $C$. albicans cells, only MAbs E2-9 and M2-4 in combination are able to convey full 100 $\%$ protection when is given only once. The same complete protection can be accomplished by the repeated administration of each MAb for two- weeks. It is obvious that the efficacy of immunoprophylaxis is augmented when MAbs E2-9 and M2-4 used in combination. In conclusion, the combination of MAb E2-9 and MAb M2-4, each of which recognizes a unique cell surface peptide epitope, increased the protection efficacy. We are now in the throes of determining the mechanism by which MAbs are protective. This issue is complex because antibodies against Candida may exert their protection by a variety of different actions [24-30]. Since each MAb recognizes unrelated peptide from different cell wall proteins, it is possible that different mechanisms of protection led to an additive, even possible a synergistic effect when the antibodies were used in combination to prevent or even treat the fungal disease. These possibilities are the subject of our on-coming experiments. The MAb combination may also have potential to enhance therapeutic efficacy of antifungal agent, which could lead to a reduction of the drug needed for treatment to non-toxic levels [30].

Regardless of the mechanism of protection afforded by antibodies specific to the Fba and Met6 peptides, the fusion of the two protective peptides into a double chimeric peptide vaccine will provide the host with two chemically distinct epitope targets to mount an immunologic response. The increasing importance of severe invasive fungal infections and the need for new treatment options for these infections have fuelled interest in development of immunoprophylaxis approaches for prevention of invasive mycoses. The demonstration that double chimeric peptide vaccine Fba-Met6, as well as two protective MAbs E2-9 \& M2-4 in combination, can confer complete protection against invasive candidiasis in animal models will establish the foundation for novel strategies for the control of this disease in humans.

\section{Acknowledgements}

We thank the Research Institute for Children (RIC) at Children's Hospital in New Orleans for support of our research. The author also thanks Dr. Jim Cutler for his inspiration and advice.

\section{References}

1. Dimopoulos G, Ntziora F, Rachiotis G, Armaganidis A, Falagas ME (2008) Candida albicans versus non-albicans intensive care unit-acquired bloodstream infections: differences in risk factors and outcome. Anesth Analg 106: 523-529, table of contents.

2. Viudes A, Pemán J, Cantón E, Ubeda P, López-Ribot JL, et al. (2002) Candidemia at a tertiary-care hospital: epidemiology, treatment, clinical outcome and risk factors for death. Eur J Clin Microbiol Infect Dis 21: 767-774.

3. Krcmery V, Barnes AJ (2002) Non-albicans Candida spp. causing fungaemia: pathogenicity and antifungal resistance. J Hosp Infect 50: 243-260.

4. Cassone A (2013) Development of vaccines for Candida albicans: fighting a skilled transformer. Nat Rev Microbiol 11: 884-891.

5. Xin H, Dziadek S, Bundle DR, Cutler JE (2008) Synthetic glycopeptide vaccines combining beta-mannan and peptide epitopes induce protection against candidiasis. Proc Natl Acad Sci U S A 105: 13526-13531.

6. Xin H, Cutler JE (2011) Vaccine and monoclonal antibody that enhance mouse resistance to candidiasis. Clin Vaccine Immunol 18: 1656-1667.

7. .Han Y, Cutler JE (1995) Antibody response that protects against disseminated candidiasis. Infect Immun 63: 2714-2719.

8. Han Y, Riesselman MH, Cutler JE (2000) Protection against candidiasis by an immunoglobulin G3 (IgG3) monoclonal antibody specific for the same mannotriose as an IgM protective antibody. Infect Immun 68: 1649-1654.

9. Son YI, Egawa S, Tatsumi T, Redlinger RE Jr, Kalinski P, et al. (2002) A novel bulk-culture method for generating mature dendritic cells from mouse bone marrow cells. J Immunol Methods 262: 145-157.

10. Pitarch A, Abian J, Carrascal M, Sánchez M, Nombela C, et al. (2004) Proteomics-based identification of novel Candida albicans antigens for diagnosis of systemic candidiasis in patients with underlying hematological malignancies. Proteomics 4: 3084-3106.

11. Clancy CJ, Nguyen ML, Cheng S, Huang H, Fan G, et al. (2008) Immunoglobulin $\mathrm{G}$ responses to a panel of Candida albicans antigens as accurate and early markers for the presence of systemic candidiasis. J Clin Microbiol 46: 1647-1654.

12. Spellberg B (2011) Vaccines for invasive fungal infections. F1000 Med Rep 3: 13 .

13. Lennon-Duménil AM1, Bakker AH, Wolf-Bryant P, Ploegh HL, Lagaudrière-Gesbert C (2002) A closer look at proteolysis and MHCclass-II-restricted antigen presentation. Curr Opin Immunol 14: 15-21.

14. Han Y, Kanbe T, Cherniak R, Cutler JE (1997) Biochemical characterization of Candida albicans epitopes that can elicit protective and nonprotective antibodies. Infect Immun 65: 4100-4107.

15. Moss CX, Tree TI, Watts C (2007) Reconstruction of a pathway of antigen processing and class II MHC peptide capture. EMBO J 26: 2137-2147.

16. Vogel FR, Alving (2002) Progress in immunologic adjuvant development. 39-43,

17. Jackson DC, Purcell AW, Fitzmaurice CJ, Zeng W, Hart DN (2002) The central role played by peptides in the immune response and the design of peptide-based vaccines against infectious diseases and cancer. Curr Drug Targets 3: 175-196.

18. Gu Y, Wei J, Yang J, Huang J, Yang X, et al. (2013) Protective immunity against Trichinella spiralis infection induced by a multi-epitope vaccine in a murine model. PLoS One 8: e77238.

19. Tam JP (1988) Synthetic peptide vaccine design: synthesis and properties of a high-density multiple antigenic peptide system. Proc Natl Acad Sci U S A 85: 5409-5413.

20. Cassone A, De Bernardis F, Torososantucci A (2005) An outline of the role of anti-Candida antibodies within the context of passive immunization and protection from candidiasis. Curr Mol Med 5: 377-382.

21. Casadevall A, Dadachova E, Pirofski LA (2004) Passive antibody therapy for infectious diseases. Nat Rev Microbiol 2: 695-703.

22. Pohl MA, Rivera J, Nakouzi A, Chow SK, Casadevall A (2013) Combinations of monoclonal antibodies to anthrax toxin manifest new properties in neutralization assays. Infect Immun 81: 1880-1888. 
Citation: Xin H (2014) Double Chimeric Peptide Vaccine and Monoclonal Antibodies that Protect Against Disseminated Candidiasis . J Vaccines Vaccin 5: 241. doi:10.4172/2157-7560.1000241

Page 9 of 9

23. Casadevall A, Pirofski LA (2007) Antibody-mediated protection through cross-reactivity introduces a fungal heresy into immunological dogma. Infect Immun 75: 5074-5078.

24. Torosantucci A, Chiani P, Bromuro C, De Bernardis F, Palma AS, et al (2009) Protection by anti-beta-glucan antibodies is associated with restricted beta-1,3 glucan binding specificity and inhibition of fungal growth and adherence. PLoS One 4: e5392.

25. Bromuro C, Romano M, Chiani P, Berti F, Tontini M, et al. (2010) Betaglucan-CRM197 conjugates as candidates antifungal vaccines. Vaccine 28: 2615-2623.

26. De Bernardis F, Boccanera M, Adriani D, Spreghini E, Santoni G, et al (1997) Protective role of antimannan and anti-aspartyl proteinase antibodies in an experimental model of Candida albicans vaginitis in rats. Infect Immun 65: 3399-3405.
27. Torosantucci A, Bromuro C, Chiani P, De Bernardis F, Berti F, et al. (2005) A novel glyco-conjugate vaccine against fungal pathogens. J Exp Med 202: 597-606.

28. Brena S, Omaetxebarría MJ, Elguezabal N, Cabezas J, Moragues MD, et al. (2007) Fungicidal monoclonal antibody C7 binds to Candida albicans Als3. Infect Immun 75: 3680-3682.

29. Moragues MD, Omaetxebarria MJ, Elguezabal N, Sevilla MJ, Conti S, et al. (2003) A monoclonal antibody directed against a Candida albicans cell wall mannoprotein exerts three anti-C. albicans activities. Infect Immun 71: 5273-5279.

30. Chianese-Bullock KA, Lewis ST, Sherman NE, Shannon JD, Slingluff CL Jr (2009) Multi-peptide vaccines vialed as peptide mixtures can be stable reagents for use in peptide-based immune therapies. Vaccine 27: 1764-1770. 